\title{
400 PERSISTENCE AND TISSUE DISTRIBUTION OF AGENT- 797 - A NATIVE ALLOGENEIC INKT CELL-THERAPY DRUG PRODUCT
}

${ }^{1}$ Marco Purbhoo*, ${ }^{1}$ Burcu Yigit*, ${ }^{1}$ Darrian Moskowitz, ${ }^{2}$ Min Lim, ${ }^{2}$ rina Shapiro,

${ }^{2}$ Ayat Alsaraby, ${ }^{1}$ Xavier Michelet, ${ }^{1}$ Marc Van Dijk. ${ }^{1}$ Mink Therapeutics, Abingdon, UK; ${ }^{2}$ Agenus, Lexington, MA, USA

Background Invariant Natural Killer T (iNKT) cells are key effectors and regulators of immune responses, making them an ideal immunotherapy. There is a paucity of evidence describing the persistence and trafficking of these cells in humans to inform the optimal clinical application. Here, we describe the development of a murine Xenograft model for the study of an unmodified human iNKT cell therapy (Agent-797) and present data on the persistence and tissue distribution of human iNKT cells in this model. We further describe the development and validation of a digital PCR-based methodology to track unmodified allogeneic human iNKT cells in blood and tissue and present exploratory clinical data on iNKT cell persistence in patients with cancer and viral ARDS treated with Agent-797.

Methods Persistence and tissue distribution of ex-vivo expanded human iNKT cells was investigated in immune compromised mice (NOG), as well as in NOG mice expressing human IL15 (NOG-hIL15), a key cytokine promoting iNKT cell survival. Persistence of iNKT cells was determined over a 35-day period, with takedowns on day 1, 7, 14, 21 and 35. iNKT cells were phenotyped for activation markers by flow cytometry. An assay based on Imegen Quimera digital PCR technology was developed and validated to quantify human iNKT in an allogeneic setting. We employed this assay to measure persistence of Agent-797 drug product in patients participating in clinical trials using iNKT cell-based immunotherapy in viral ARDS (NCT04582201) or multiple myeloma (NCT04754100).

Results Human IL15 was essential for the engraftment and persistence of human iNKT cells in NOG mice. Following injection, iNKT cells located to the blood, lung, liver, spleen, and bone marrow. iNKT cells persisted most prominently in bone marrow, where they demonstrated an activated phenotype. In mice challenged with hematological tumor cells (ALL cell line NALM6 expressing CD1d) persistence of iNKT cells in blood was prolonged. Initial data from human trials confirmed rapid translocation from peripheral blood of this tissue resident immune cell population following infusion of Agent797.

Conclusions We established a murine xenograft model and digital PCR-based methodology to characterize the persistence, trafficking, and efficacy of native allogeneic human iNKT cellbased products. Our models recapitulated the human iNKT distribution and demonstrated iNKTs induced preclinical efficacy in a tumor model. We further successfully developed a validated methodology to track unmodified allogeneic iNKT cells in humans.

Trial Registration NCT04582201 and NCT04754100

Ethics Approval All procedures performed in studies involving human participants were in accordance with the ethical standards of the institutional and/or national research committee and with the 1964 Helsinki declaration and its later amendments or comparable ethical standards 